\title{
Laparoscopic surgery for endometriosis-associated infertility: a pathophysiologic approach
}

\author{
Geetu Pahlajani • Tommaso Falcone
}

Received: 10 December 2009 / Accepted: 25 January 2010/Published online: 9 March 2010

(C) Springer-Verlag 2010

\begin{abstract}
Endometriosis has been one of the most confusing gynecological diseases since it was first described. Whereas there is a reasonable body of evidence in literature to demonstrate an association between endometriosis and infertility, a definite cause and effect relationship has not been established. The mechanism by which endometriosis causes infertility remains an enigma. Virtually every aspect of reproduction in women with endometriosis has been investigated and purported to be impaired. Impairment of implantation and pregnancy rates seems to affect women with endometriosis. Whether this is due to poor quality embryos derived from impaired oocytes or endometrial defects or both has been argued. Structural abnormalities of the uterine wall and tube in women with endometriosis have also been described by other researchers. Adding more confusion to this topic is the altered immune function and the peritoneal environment and their detrimental effects on the sperm motility and morphology. This uncertain pathophysiology has resulted in the lack of consensus on the treatment of
\end{abstract}

\footnotetext{
G. Pahlajani $(\bowtie)$

Cleveland Clinic,

Main Campus, OBGYN,

Cleveland, OH, USA

e-mail: pahlajg@ccf.org

T. Falcone

Cleveland, $\mathrm{OH}$, USA

T. Falcone

Office of Professional Staff Affairs,

9500 Euclid Ave-A81,

Cleveland, $\mathrm{OH}, \mathrm{USA}$
}

Obstetrics, Gynecology and Women's Health Institute, endometriosis-associated infertility. The aim of this review is to describe the current pathophysiology of endometriosis-related infertility, how laparoscopic surgery may influence fertility rates.

Keywords Laparoscopy · Endometriosis · Infertility · Pathophysiology

\section{Introduction}

Infertility is a distressing symptom associated with endometriosis, and the exact mechanism and optimal choice of management in the context of this disease remains obscure. In spite of a great deal of effort about the pathogenesis of infertility in endometriosis, it is still not clear how endometriosis compromises fertility. Although it is generally accepted that endometriosis is related to infertility, the mechanism underlying this effect and its impact on fecundity are less clear. This makes treatment very difficult since surgery is directed at the peritoneal lesions which may not be the only the factor affecting fertility. Fecundity is defined as the probability of a woman giving live birth in a given month and ranges from 0.15 to 0.20 in normal couples and decreases to 0.02 to 0.10 in untreated women with endometriosis $[1,2]$. Three-year cumulative pregnancy rates were found to be lower in women with endometriosis $(36 \%)$ as compared with women with unexplained infertility (55\%) [3]. Numerous studies have indicated poor pregnancy outcomes in endometriosis to be associated with poor sperm function, poor ovarian reserve, lower oocyte/ embryo quality, decreased endometrial receptivity, and impaired implantation. In this review article, we discuss the existing evidence of the effects of endometriosis on fertility. 


\section{Mechanism of infertility in women with endometriosis}

Endometriosis and the alterations in pelvic anatomy

Distortion of the uterotubal anatomy has been proposed as one of the mechanisms of reduced fertility in patients with endometriosis. Adhesions may cover or distort the anatomy of the fallopian tubes and ovaries, thereby impeding pickup of oocytes by the fimbriae of the fallopian tube. Kissler et al. in 2006 [4] reported that endometriosis leads to a significant restriction in uterotubal transport capacity. They concluded that impeded hyperperistalsis and dysperistalsis in uterotubal transport associated with endometriosis may be the reason of infertility in these women [4]. Severe endometriosis is also associated with pelvic adhesions and a distortion of pelvic anatomy leading to a possible mechanic or anatomic disturbance of fertility [5,6]. Another factor distorting the pelvic anatomy and thus affecting fertility are large endometriomata seen in patients with extensive endometriosis. Somigliana et al. [7] in their study found that just the mere presence of an endometrioma will decrease the ovarian responsiveness to stimulation in invitro fertilization (IVF) cycles as compared with contralateral intact ovaries. They concluded that, especially in women with larger endometriomas, this difference was more evident [7]. Milingos et al. in a study found that laparoscopic removal of these large endometriomata significantly improved the fertility outcomes [8]. Therefore, diminished pregnancy rates in endometriosis due to adhesions and impaired uterotubal transport even with patent fallopian tubes and normal semen quality may be one of the factors of sub-fertility in these patients.

Endometriosis and the sperm function

The endometriosis-associated immunoinflammatory changes may have some adverse effects on the spermatozoa since these cells have to stay for some time in the female genital tract which is bathed by peritoneal fluid. Mansour et al. studied the correlation of endometriosis and sperm damage and found positive relation between sperm damage, stage of endometriosis, and duration of infertility [9]. The same group also studied the impact of peritoneal fluid from women with endometriosis on sperms and found a significant increase in sperm DNA damage (Fig. 1). They proposed this as one of the mechanisms of infertility in patients with endometriosis [10]. Whether this damage is due to oxidative stress, cytokines, or nitric oxide on the sperms has been debated. There is compelling evidence in literature that oxidative stress (OS) is increased in the peritoneal fluid $(\mathrm{PF})$ in patients with endometriosis. This is thought to be due to increased production of reactive oxygen species (ROS), defective antioxidant defense, or

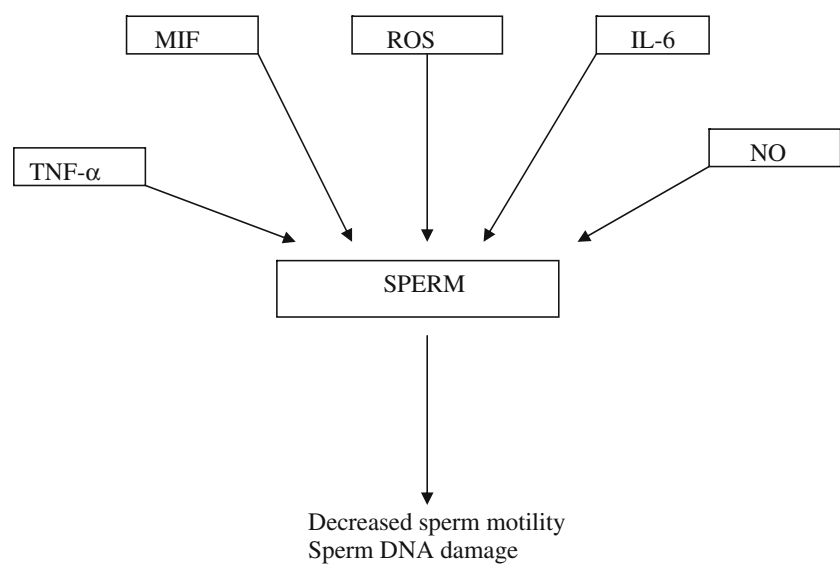

Fig. 1 Diagram: factors affecting sperm function in endometriosis

both $[11,12]$. Spermatozoa are susceptible to damage by ROS due to limited antioxidant defense and high content of polyunsaturated fatty acids in their membranes [12]. ROS produces infertility by damaging the sperm plasma and acrosomal membrane by lipid per oxidation, decreasing sperm motility, and the ability of the sperm to bind and penetrate the oocyte and causing DNA damage leading to defective embryo [13-15].

The role of cytokines has been implicated in the pathogenesis of endometriosis and related infertility. Interleukin-6 (IL-6) and its soluble receptor (sIL-6R) have been reported to be higher in the peritoneal fluid of infertile patients with endometriosis [16, 17]. Yoshida et al. proposed that combination of IL- 6 and sIL-6R may be associated with gp-130 expressed in the sperm and thus reducing sperm motility and contributing to the pathogenesis of endometriosis-related infertility [18]. Macrophage inhibiting factor (MIF), another multifunctional cytokine has been found to be significantly increased in the peritoneal fluid and endometrium of women with endometriosis [19, 20]. Carli et al. [21] in 2007 studied the dosedependent effect of MIF on sperm capacitation and concluded that high amounts of MIF had adverse effects on capacitation and sperm motility. They suggested that increased levels of MIF in women with endometriosis may play a role in endometriosis-associated infertility [21]. Tumor necrosis factor alpha (TNF- $\alpha)$, a potent cytokine has also been found to be increased in patients with endometriosis and has been proposed as a sensitive marker for the non-surgical diagnosis of endometriosis [16, 22]. Said et al. in their study reported that pathological concentrations of TNF- $\alpha$ can result in loss of sperm motility, plasma membrane functional integrity, as well as DNA fragmentation [23]. The same group implicated that infliximab, an anti-inflammatory drug, may be used to reverse the toxic effects of TNF- $\alpha$ on spermatozoa and thus help treat female infertility in endometriosis patients [24]. 
Another proposed mechanism of infertility in endometriosis is due to deleterious effect of nitric oxide (NO) on the sperms. Osborn et al. in a study found that peritoneal macrophages from women with endometriosis-associated infertility express higher levels of inducible nitric oxide synthase and produce more NO than fertile controls, and high levels of NO have a deleterious effect on sperm motility and function $[25,26]$. Moreover, Lampiao et al. found that TNF- $\alpha$ and IL- 6 have detrimental effect on the spermatozoa via an increase in NO production [27].

\section{Endometriosis and ovarian function}

Good quality embryos originate from good quality oocytes, which in turn originate from follicles with an adequate environment conditioned by the follicular fluid and the neighboring cells, which are able to influence their progression [28]. Altered intrafollicular microenvironment influences the oocyte growth and development via a close relationship with the granulose cells and other ovarian cell types. Mansour et al. found significant DNA damage in the oocytes that were incubated in the peritoneal fluid of patients with endometriosis as compared with normal controls. The extent of damage was related to the duration of exposure to the PF of endometriosis [29]. Whether this damage to oocytes is due to hormonal factors, cytokines, leucocytes, apoptic bodies, or OS has been studied and documented in literature. Studies have shown consistent evidence of subtle pituitary-ovarian dysfunction associated with endometriosis, linked to considerable impairment of oocyte fertilizing ability and a probable impairment of granulosa cell steriodogenesis. Impaired follicular growth, reduction in circulating estradiol concentration during the pre-ovulatory phase and of estradiol and progesterone during the early luetal phase and disturbed luteinizing hormone surge pattern have been documented as a cause of poor oocyte quality and sub-fertility in endometriosis [30]. Similarly, other authors recorded zygote formation and embryo development in vitro and reported that the percentage of abnormal embryos were higher in endometriotic patients [31]. Diaz et al. [32] in their study implicated that even severe endometriosis did not affect the implantation rate, and the poor pregnancy outcomes were probably due to poor quality oocytes or embryos. They found no change in the in-vitro fertilization-embryo transfer (IVFET) outcomes in endometriotic patients receiving donor oocytes [32]. However, contradictory results have been shown by other groups which found no change in granulose luteal cell steriodogenesis, normal maturation of oocytes, and embryo in the altered hormonal milieu [33, 34].

Apart from the endocrine environment, various paracrine factors secreted by the granula cell and present in the follicular fluid could interfere with the oocyte development.
In a study done by Pellicer et al. [35], serum IL-6 were found to be increased in natural cycle in women with endometriosis and decreased in stimulated cycles in IVF. IL-6 was also increased in the follicular fluid of women with endometriosis and released in higher amounts by granulosa cells in these women. In addition, vascular endothelial growth factor (VEGF) has been found to be in lower concentrations in endometriotic patients [35], and elevated VEGF has also been correlated in IVF with good follicular vascularization and health [36]. Garrido et al. postulated that infertility in patients with endometriosis may be related to these changes in the follicular environment leading to altered oocytes which in turn results in poor quality of embryos [37]. Another study found that the granula cells from endometriotic patients had an increased production of IL- $1 \beta$, IL- 6 , IL- 8 , and TNF- $\alpha$ compared with healthy women, although the increase for TNF- $\alpha$ was more significant. Furthermore, HCG suppressed these cytokines in both the endometriotic and healthy women [38]. Whether these changes in the follicular environment causes disturbances in the synchronization of oocyte maturation, ovulation, and uterine receptivity affecting the fertilization need to be further investigated.

Another interesting theory postulated is the role of ovarian leukocytes on oocyte maturation and growth. Leukocyte present within the ovary may constitute as potential in situ modulators of ovarian function that act through the local secretion of numerous cytokines [39]. Lachapelle et al. found an increase in natural killer (NK) cells and monocytes (CD4) in endometriotic patients [40]. In contrast, another study using flow cytometry to determine the presence of total leukocytes and their subsets in the follicle from patients with endometriosis and healthy controls found no changes in the leukocyte portions and activity [41]. Establishing the definite role of leukocytes in oocyte maturation is difficult due to the aforementioned conflicting studies. The role of apoptic cells on folliculogenesis in endometriosis has also been studied. Toya et al. concluded that in the granulosa cells of patients with endometriosis, an increased incidence of altered cell cycle and apoptic bodies are found as compared with healthy controls [42], and it has been found that a lower incidence of apoptic bodies in individual follicles has been associated with better outcome of oocyte quality [43].

The role of OS has been implicated in various aspects of female reproduction and infertility including oocyte viability and its ability to fertilize with the spermatozoa [44, 45]. Increased production of ROS by peritoneal macrophages and diminished peritoneal fluid antioxidant results in OS in the peritoneal microenvironment of patients with endometriosis [46]. OS leads to localized pelvic inflammatory reaction resulting in increased concentrations of cytokines, growth factors, and other inflammatory mediators which in 
turn induce lipid peroxidation, resulting in the formation of cytotoxic lipid peroxides and DNA damage [46, 47]. One of the most popular markers for oxidative DNA damage and OS is 8-hydroxy-deoxyguanosine which is found to be increased in infertile patients with endometriosis than infertile patients with other causes [48]. These changes caused by OS might lead to rapid cellular death and oocyte degeneration.

\section{Endometriosis and fertilization}

The effect of follicular fluid on the binding of human spermatozoa to the zona pellucida has been investigated in women with endometriosis. Qiao et al. suggested that patients with endometriosis had a stronger sperm-zona binding inhibitory effect in their follicular fluid than patients without endometriosis which may contribute to impairment of gamete interaction [49]. Additionally, sperm mixed with peritoneal fluid from women with endometriosis has been shown to perform poorly on a zona-free hamster egg sperm penetration assay [50]. Barbara et al. found that macrophage secretory products, particularly TNF- $\alpha$, may interfere sperm-zona pellucida binding and may lead to infertility in women with endometriosis [51]. Interestingly, a retrospective study of the impact of endometriosis on IVF outcomes found that patients with stages 3 and 4 endometriosis had a poorer IVF-ET outcome than patients with tubal infertility. The authors suggested that maternal serum in patients with endometriosis adversely affects the fertilization rate, and this reduction in fertilization may be due to either decreased oocyte quality or the detrimental effect of the cumulus-corona complex [52]. However, Olivennes et al. [53] found no difference in the pregnancy outcome in patients with endometriosis and tubal infertility. Pregnancy rates in pure endometriosis patients without other concomitant infertility factors were found to be similar to those patients with tubal infertility [53].

Endometriosis and implantation

There have been studies in literature suggesting impairment of implantation in patients with endometriosis [54]. Whether this defect is due to abnormal endometrium or defective embryos is debated (Table 1). Minici et al. suggested that the milieu surrounding the uterine cavity, particularly increased peritoneal levels of TNF- $\alpha$, compromise the normal decidualization required for optimal implantation [55]. Lessey et al. in their study reported a reduced expression of $\alpha \mathrm{v} \beta 3$ integrin, a cellular adhesive molecule in the endometrium of patients with endometriosis during the window of implantation [56]. Another study has shown that, in women with endometriosis, a significant increase in endothelial nitric oxide synthase during the midluteal phase was concomitant with a drastic decrease in adhesion molecule $\alpha \mathrm{v} \beta 3$. Such imbalance may strongly contribute to implantation defects [57]. In contrast to this, Hii et al. found no difference in the glandular expression of $\alpha \mathrm{v} \beta 3$ integrin between endometriosis patients and normal controls [58]. In an interesting study, Matsuzaki demonstrated that HOXA-10m-RNA and protein expression levels in the endometrial stromal cells were significantly lower during the window of implantation in patients with endometriosis probably leading to infertility [59]. Hugo et al. in their study found that altered aromatase expression in the eutopic endometrium of women with endometriosis may hamper the ovum nidation, thus causing infertility [60]. However, Kao et al. [61] suggested alteration in candidate genes contributing to implantation failure. Their data supported that the dysregulation of selected genes such as BSEP, C4BP, IL-15, etc. may promote an inhospitable environment for embryonic implantation [61]. In a recent study, expression of EMX2, a transcription factor necessary for reproductive tract development negatively regulated by HOXA10 gene, was found to be altered. The authors suggested that high EMX2 and low HOXA10 in the periimplantation endometrium of patients with endometriosis alter the pattern of target gene expression, thus inhibiting implantation [62]. Another study indicated the possibility that infertile women with endometriosis have abnormal production of IL-11 and leukemia inhibitory factor which may contribute to altered uterine receptivity and thus leading to infertility [63]. All of the abovementioned studies found a defect in the endometrium impairing the implantation in endometriosis patients. In contrast, other authors have given impaired oocyte/embryo quality as an alternative explanation for decreased implantation. Sung et al. in their study concluded that endometriosis in oocyte recipients does not lower implantation, and the adverse effect of endometriosis on implantation is probably due to the abnormal oocyte quality [64].This finding was recently confirmed by Diaz et al. [32] in a study in which oocytes from healthy donors were divided in the same cycle between different receivers with and without endometriosis. Recipients with stages 3 and 4 endometriosis had the same implantation rate as controls [32].These studies show that endometriosis does not impair implantation in oocyte recipients, suggesting a defective quality of oocytes/embryo as the cause of infertility in women with endometriosis.

Endometriosis and immune-endocrine interaction

There is considerable evidence in literature indicating the association of humoral and cell-mediated immunity with endometriosis. The peritoneal fluid of women with endo- 
Table 1 Endometrial factors impairing implantation

\begin{tabular}{lll}
\hline Factor & Level of the factor & Reference \\
\hline TNF- $\alpha$ & Increased & Minci et al. [55] \\
$\alpha \mathrm{v} \beta 3$ integrin & Decreased & Lessey et al. [56] \\
e-NOS & Increased & Khorram et al. [57] \\
HOXA-10m-RNA & Decreased & Matsuzaki et al. [59] \\
Aromatase expression & Increased & Hugo et al. [60] \\
BSEP, C4BP,IL-15 genes & Dysregulated & Kao et al. [61] \\
EMX2 & Increased & Daftary et al. [62] \\
IL-11, LIF & Decreased & Dimitriadis et al. [63] \\
\hline
\end{tabular}

metriosis contains an increased number of immune cells which facilitate the development of endometriosis rather than inhibiting it [65]. Autoantibodies, several cytokines, and growth factors display increased levels in the peritoneal fluid of women with endometriosis. Mathur et al. were the first to report $\operatorname{IgG}$ and $\operatorname{IgA}$ antibodies in the vaginal and cervical secretions of women with endometriosis [66]. Gleicher et al. [67] in their study found a wide variety of autoantibodies in endometriosis patients suggesting polyclonal activation of $\mathrm{B}$ cell, characteristic of autoimmune disease. They argued that ectopic endometrium might induce an autoimmune response and contribute to infertility associated with endometriosis [67]. Furthermore, treatment with danazol and GnRH analog suppresses the antiendometrial antibodies associated with endometriosis [68]. Another study showed that infertile women with endometriosis had various kinds of autoantibodies, especially against phospholipids in serum and the peritoneal fluids that could reduce the success of spontaneous and artificial implantation [68]. Although these data suggest that autoantibodies may play a role in infertility associated with endometriosis, the importance of autoimmunity in pathogenesis of infertility in these patients needs to be further explored.

Cytokines are diverse proteins that play a central role in regulating cellular activity. Several studies have reported a potential link between cytokines and the pathogenesis of endometriosis. Interluekin-1 (IL-1) is one of the major proinflammatory cytokines found in the peritoneal fluid of women with endometriosis, and studies have found that IL1 may play a role in the infertility associated with endometriosis [69, 70]. IL-1 has been found to inhibit mouse embryo development, impairing the capacity of sperm to penetrate the oocyte without altering the sperm motility [71, 72]. In a recent study, it was suggested that an imbalance between IL-1 and its inhibitory soluble IL-1 receptor type 2 levels in peritoneal fluid of women with endometriosis may cause a defect in the local control of IL1 and may be involved in the pathophysiology of endometriosis and its related infertility [73]. IL-6, another pleiotropic cytokine, has been associated with reproductive physiology and found to be increased in the peritoneal fluid of women with endometriosis [16, 17]. The elevated levels of IL- 6 has been associated with poor sperm motility and altered follicular functions [18, 35]. Deura et al. in their study showed that IL-6 suppressed estrogen production and aromatase activity in the granula cell line and may be associated with infertility with endometriosis [74]. Another cytokine IL-17 has been found to induce IL- 6 and TNF- $\alpha$ in the macrophages, which play a crucial role in the pathogenesis of endometriosis and infertility [75]. Zhang et al. in their study found that IL-17 was significantly higher in patients with mild/minimal endometriosis and also when endometriosis was associated with infertility [76]. TNF- $\alpha$ is another cytokine that plays a key role in the multitude of inflammatory processes. TNF- $\alpha$ has been implicated in the pathophysiology of endometriosis and its associated infertility. It affects sperm motility and also shows embryotoxic effects [23, 55].

RANTES (regulated on activation, normal T-cell expressed and secreted) is another cytokine chemoattractant for monocytes as well as memory $\mathrm{T}$ cells and eosinophils. The level of RANTES is found to be increased in the peritoneal fluid of women with endometriosis, and its level correlates with the severity of disease [77]. Xu et al. [78] found poor IVF outcomes in endometriosis-related infertility than in tubal infertility. They concluded that elevated follicular fluid RANTES evokes an altered inflammatory milieu within the follicular fluid environment leading to poor oocyte quality [78]. MIF is a multifunctional cytokine that regulates immune response, cell proliferation, and angiogenesis. Morin et al. found elevated levels of MIF in the peripheral blood of women with endometriosis and suggested that MIF may adversely affect fertility in these women [79]. Monocyte chemo-tactic protein 1 is a $\beta$ chemokine produced mainly by the monocytes, and macrophages have been found to be increased in infertile patients with endometriosis [80]. Complement, another component of the humoral immunity, has been found to be altered in endometriosis. Kabut et al. in their study found increased concentrations of $\mathrm{C} 3 \mathrm{c}$ and decreased concentration of $\mathrm{iC} 3 \mathrm{~b}$ in the $\mathrm{PF}$ of women with 
endometriosis, both of which are derivatives of $\mathrm{C} 3$ component of the complement cascade [81]. A higher concentration of $\mathrm{iC} 3 \mathrm{~b}$, produced from the oviductal $\mathrm{C} 3 / \mathrm{C} 3 \mathrm{~b}$ has been found to enhance the development of blastocyst and also stimulate embryo development [82]. This decreased $\mathrm{iC} 3 \mathrm{~b}$ found in the PF of women with endometriosis could lead to infertility. There is a controversy on the role of increased level of leptin in endometriosis-related infertility. Leptin, a product of the obese gene, is a cytokine similar to various members of the interleukin family. Barcz et al. suggested that the increased levels of leptin in peritoneal fluid in women with endometriosis may be associated with infertility [83]. On the other hand, Bedaiwy et al. in their study found that increased peritoneal fluid leptin levels may be associated with pain but not infertility in patients with endometriosis [84].

Endometriosis is associated with changes in cellmediated immunity as well. Various components of cellular immunity have been found to be altered in endometriosis. Peritoneal macrophages are the major resident cells in the peritoneal cavity, and their number, concentration, and activity are higher in patients with endometriosis than controls [85]. NK cell activity in women with endometriosis has been shown to have a decreased cytotoxic activity, which indicates alteration in immune response, and coexisting with endometrial abnormalities [86]. These changes in the peritoneal environment may also have a role in endometriosis-related infertility [87]. Moreover, danazol and GnRH analogs, which are commonly used for treatment of endometriosis down-regulate humoral and cellular immunity concomitantly with their effect on endometriotic implants [88].

Hyperprolactinemia has been reported to exist in patients with endometriosis-related infertility, but the role of prolactin (PRL) in infertile women with endometriosis is less clear [89]. In a study, it was suggested that altered PRL secretion and decreased serum estradiol after thyrotrophinreleasing hormone administration in infertile women with endometriosis was strongly related to a dysfunction of the hypothalamic-hypophyseal-ovarian axis and could be the cause of infertility in these patients [90]. Matrix metalloproteinase-2 (MMP-2) has emerged as one of the key participants in the adhesion and proliferation of shed menstrual tissue in the pathogenesis of endometriosis [91]. Estradiol has been shown to up-regulate the MMP-2 action leading to formation of endometriosis, and progesterone has been associated with down-regulation of MMP-2 action inhibiting the development of endometriosis [92]. MMP-2 has shown to have a role in ovulation and luteal function [93].

There is clear evidence from the above discussion on the interaction between immune mediators and hormones on the development of endometriosis and its associated infertility. A better understanding of these interactions will set the stage for immune-targeted therapies not only for the management of endometriosis but also its associated infertility.

Endometriosis and role of laparoscopy in management of infertility

Although there is an association between endometriosis and infertility, the discussion about exact causal relationship is still ongoing. Nevertheless, laparoscopy has been used for the diagnosis, staging, and treatment of endometriosis. Visual look of the disease through laparoscopy has become an integral part of management of infertility in endometriosis. The effect of destruction of peritoneal endometriosis (ASRM stage I-II) by laparoscopy on pregnancy rates in infertile women has been debated [94]. It is unclear how removing or ablating a lesion by laparoscopy will improve the alterations described above. Two randomized controlled trials did not agree on the effect of laparoscopic ablation or excision of endometriotic lesions on pregnancy rates. The larger study, by the Canadian Collaborative Group on Endometriosis, showed that excision or laparoscopic ablation of minimal and mild endometriosis increased ongoing pregnancy rates in infertile women (OR 1.95, 95\% CI 1.183.22) [95]. The number needed to treat in the Canadian study was nine. A smaller trial by an Italian group failed to show significant impact on the live birth rate (OR 0.85 , 95\% CI 0.32-2.28) [96]. However, when these two results were combined, the pooled odds ratio showed a significant improvement in live birth rate following surgical treatment (OR 1.64, CI 1.05-2.57) [97]. If we combine the Canadian and the Italian studies, the number needed to treat is 12 . Thus, there is reasonable evidence to treat minimal and mild endometriosis to improve the subsequent fertility outcome. Laparoscopic treatment of mild endometriosis either by excision or coagulation was shown to have no influence on the pregnancy rate and had similar outcomes [98].

An interesting study by Bedaiwy et al. on the impact of surgery on IVF outcome found no improvement in pregnancy outcomes [99]. In light of the complex pathophysiological changes taking place in the peritoneum in endometriosis, surgical removal of lesions may therefore not necessarily have a substantial impact on fertility.

Similarly, laparoscopic treatment of ovarian endometriosis (ASRM III-IV) showed varied outcomes and the pregnancy rates ranged from $30 \%$ to $67 \%[100,101]$. Despite the possible risk of loss of ovarian tissue and disruption of blood supply leading to ovarian damage, literature shows increased pregnancy rates following laparoscopic treatment of endometriomas [102]. In a recent Cochrane review, it was concluded that the excision of the 
endometriomas improves the subsequent spontaneous pregnancy rates in comparison to drainage and ablation of the endometrioma [103]. The removal of endometriomata may be associated with risk of damage to ovarian function. Ovarian cystectomy may cause resection of the healthy ovarian cortex with follicles rather than excision of intraovarian cyst. Somigliana found fewer follicles in response to gonadotropin stimulation after laparoscopic removal of endometriomata thereby altering the ovarian response during IVF [104]. Additionally, adverse changes in the ovarian artery blood flow have been reported following laparoscopic stripping [105]. Nonetheless, careful removal of endometriomas will improve spontaneous fertility substantially and should be removed.

Recently, interest has been shown on the influence of laparoscopic treatment of bowel endometriosis on postoperative fertility. Stepniewska et al. [106] in one of the largest series retrospectively looked at the effect of laparoscopic surgery for endometriosis with colorectal segmental resection on subsequent fecundity rate. They concluded that complete removal of endometriosis with bowel segmental resection had better post-operative fertility than removal of endometriosis without bowel resection [106]. Ferrero et al. in their study suggested that although spontaneous pregnancy may occur after bowel resection, these patients may require infertility treatment, especially women $\geq 35$ years [107]. Vercellini et al. [108] studied the role of conservative surgery for rectovaginal endometriosis on the fertility outcome. Their group concluded that, in patients with no other associated major infertility factors, surgical resection of rectovaginal endometriosis did not improve the pregnancy outcome [108].

Another interesting aspect is the impact of laparoscopic surgery for recurrent endometriosis on subsequent fertility. Fedele et al. [109] compared the 5-year cumulative pregnancy after laparoscopic excision of primary versus recurrent ovarian endometrioma in the same ovary as the primary cyst. The 5-year cumulative pregnancy rate was $40.8 \%$ after the first surgical procedure and $32.4 \%$ after the second procedure. The authors concluded that the effect of repetitive laparoscopic surgery is similar to that observed after first-line surgery and that the women with repetitive surgery underwent assisted reproduction techniques more frequently [109].

\section{Conclusion}

Pelvic endometriosis is frequently associated with infertility even when the affected women have functional, patent tubes. Beset with inadequate, inconclusive, and conflicting data, it remains difficult to arrive at a consensus regarding the exact mechanism of infertility in these patients. In this review, we have discussed the various possible mechanisms that can affect fertility which can later be a scope for future management of the disease. Despite the lack of firmly established causal relation between endometriosis and infertility, these developments may help improve the fertility rates in these patients. Laparoscopy still remains the gold standard for the diagnosis and management of the disease. Laparoscopic management of minimum to mild endometriosis has been shown to improve subsequent fertility outcomes, although it is unclear by how much it is improved. Laparoscopic removal of endometriomas will improve spontaneous pregnancy rates significantly. Laparoscopic excision rather than drainage and ablation of endometrioma are recommended. The role of bowel surgery in fertility outcome requires more research.

Conflict of interest There is no conflict of interest. There have been no financial interest/arrangements with one or more organizations that could be perceived as a real or apparent conflict of interest in the context of the subject of this article.

\section{References}

1. Schwartz D, Mayaux MJ (1982) Female fecundity as a function of age: results of artificial insemination in 2193 nulliparous women with azoospermic husbands. Federation CECOS. N Engl J Med 306:404-406

2. Hughes EG, Fedorkow DM, Collins JA (2001) A quantitative overview of controlled trials in endometriosis associated infertility. Fertil Steril 75(5):1042-1044

3. Akande VA, Hunt LP, Cahill DJ et al (2004) Differences in time to natural conception between women with unexplained infertility and infertile women with minor endometriosis. Hum Reprod 19:96-103

4. Kissler J, Hamscho N, Zangos S et al (2006) Uterotubal transport disorder in adenomyosis and endometriosis - a cause for infertility. BJOG 113:902-908

5. Guzick DS, Sillian NP, Adamson GD et al (1997) Prediction of pregnancy in infertile women based on the American Society for Reproductive Medicine's revised classification of endometriosis. Fertil Steril 67:822-829

6. Stovall DW, Bowser LM, Archer DF et al (1997) Endometriosisassociated pelvic pain: evidence for an association between the stage of disease and a history of chronic pelvic pain. Fertil Steril 68:13-18

7. Somigliana E, Infantino M, Benedetti F et al (2006) The presence of ovarian endometriomas is associated with a reduced responsiveness to gonadatropins. Fertil Steril 86:192-196

8. Milingos S, Kallipolitis G, Loutradis D et al (1998) Factors affecting postoperative pregnancy rate after endoscopic management of large endometriomata. Int J Gynecol Obstet 63:129-137

9. Mansour G, Goldberg J, Agarwal A et al (2007) Correlation between sperm DNA damage, stage of endometriosis and the duration of infertility. Fertil Steril 88:S205-S206, Abstracts

10. Mansour G, Aziz N, Sharma R et al (2009) The impact of peritoneal fluid from healthy women and from women with endometriosis on sperm DNA and its relationship to the sperm deformity index. Fertil Steril 92:61-67 
11. Augoulea A, Mastorakos G, Lambrinoudaki I et al (2009) The role of oxidative stress in the endometriosis related infertility. Gynecol Endocrinol 25(2):75-81

12. Langendonckt AV, Roux FC, Donnez J (2002) Oxidative stress and peritoneal endometriosis. Fertil Steril 77:861-870

13. Saleh RA, Agarwal A, Nelson DR et al (2002) Increased sperm nuclear DNA damage in normozoospermic infertile men: a prospective study. Fertil Steril 78:313-318

14. Agarwal A, Sharma RK, Natella KP et al (2006) Reactive oxygen species as an independent marker of male factor infertility. Fertil Steril 86:878-885

15. Tremellen K (2008) Oxidative stress and male infertility-a clinical perspective. Hum Reprod Updat 14:243-258

16. Bedaiwy MA, Falcone T, Sahrma RK et al (2002) Prediction of endometriosis with serum and peritoneal fluid markers: a prospective controlled trial. Hum Reprod 17:426-431

17. Harada T, Yoshioka H, Yoshida $S$ et al (1997) Increased interleukin-6 levels in peritoneal fluid of infertile patients with active endometriosis. Am J Obstet Gynecol 176:593-597

18. Yoshida S, Harada T, Iwabe T et al (2004) A combination of interleukin-6 and its soluble receptor impairs sperm motility: implications in infertility associated with endometriosis. Hum Reprod 19:1821-1825

19. Ktas R, Collette T, Metz CN et al (2002) Marked elevation of macrophage migration inhibitory factor in the peritoneal fluid of women with endometriosis. Fertil Steril 78:69-76

20. Akoum A, Metz CN, Al Akoum M et al (2006) Macrophage inhibitory factor expression in the intrauterine endometrium of women with endometriosis varies with disease stage, infertility status, and pelvic pain. Fertil Steril 85:1379-1385

21. Carli C, Leclerc P, Metz CN et al (2007) Direct effect of macrophage inhibitory factor on sperm function: possible involvement in endometriosis-associated infertility. Fertil Steril $88: 1240-1247$

22. Bullimore DW (2003) Endometriosis is sustained by tumor necrosis factor- $\alpha$. Med Hypotheses 60:84-88

23. Said TM, Sharma RK, Bedaiwy MA et al (2004) Toxicity of tumor necrosis factor (TNF)- $\alpha$ on human spermatozoa - possible role in endometriosis associated infertility. Fertil Steril 82(suppl 2):158

24. Said TM, Agarwal A, Falcone T et al (2005) Infliximab may reverse the toxic effects induced by tumor necrosis factor alpha in the human spermatozoa: an in vitro model. Fertil Steril 83:1665-1673

25. Osborn BH, Haney AF, Misukonis MA et al (2002) Inducible nitric oxide synthase expression by peritoneal macrophages in endometriosis-associated infertility. Fertil Steril 77:46-51

26. Balercia G, Moretti S, Vignini A et al (2004) Role of nitric oxide concentrations on sperm motility. J Androl 25:245-249

27. Lampiao F, Plessis SS (2008) TNF- $\alpha$ and IL-6 affect human sperm function by elevating nitric oxide production. Reprod Biomed Online 17:628-631

28. Cecconi S, Ciccarelli C, Barberi M et al (2004) Granulosa celloocyte interactions. Eur J Obstet Gynecol Reprod Biol 115 (Suppl 1):S19-S22

29. Mansour G, Agarwal A, Radwan E et al (2007) DNA damage in metaphase II oocytes is induced by peritoneal fluid from endometriosis patients. Fertil Steril 88:S299

30. Cahill DJ, Hull MGR (2000) Pituitary-ovarian dysfunction and endometriosis. Eur Soc Hum Reprod Update 6:56-66

31. Brizek CL, Schlaff S, Pellegrini VA et al (1995) Increased incidence of aberrant morphological phenotypes in human embryogenesis - an association with endometriosis. J Assist Reprod Genet 12(2):106-112

32. Diaz I, Navarro J, Blasco L et al (2000) Impact of stage III-IV endometriosis on recipients of sibling oocytes: matched casecontrol study. Fertil Steril 74:31-34
33. Garrido N, Krussel JS, Remohi J et al (2002) Expression and function of 3 beta hydroxisteroid dehydrogenase ( $3 \beta \mathrm{HSD}$ ) type II and corticosteroid binding globulin in granulose cells from ovaries of women with and without endometriosis. J Assist Reprod Genet 19:24-30

34. Pellicer A, Valbuena D, Bauset C et al (1998) The follicular endocrine environment in stimulated cycles of women with endometriosis: steroid levels and embryo quality. Fertil Steril 69:1135-1141

35. Pellicer A, Albert C, Mercader A et al (1998) The follicular and endocrine environment in women with endometriosis: local and systemic cytokine production. Fertil Steril 70:425-431

36. Van Blerkom J, Antczak M, Schrader R (1997) The developmental potential of the human oocyte is related to the dissolved oxygen content of follicular fluid: association with vascular endothelial growth factor levels and perifollicular blood flow characteristics. Hum Reprod 12:1047-1055

37. Garrido N, Navarro J, Remohi J et al (2000) Follicular hormonal environment and embryo quality in women with endometriosis. Hum Reprod Updat 6:67-74

38. Carlberg M, Nejaty J, Froysa B et al (2000) Elevated expression of tumor necrosis factor $\alpha$ in the cultured granulose cells from women with endometriosis. Hum Reprod 15:1250 1255

39. Bukulmez O, Arici A (2000) Leukocytes in ovarian function. Hum Reprod Updat 6:1-15

40. Lachapelle MH, Hemmings R, Roy DC et al (1996) Flow cytometric evaluation of leukocyte subpopulations in the follicular fluids of infertile patients. Fertil Steril 65:1135-1140

41. Garrido N, Albert C, Mercader A et al (1999) Leukocyte subpopulations in ovarian follicles in patients with endometriosis. Hum Reprod 14:227, abstract book 1

42. Toya M, Saito H, Ohta N et al (2000) Moderate and severe endometriosis is associated with alterations in the cell cycle of granulose cells in patients undergoing in vitro fertilization and embryo transfer. Fertil Steril 73:344-350

43. Nakahara K, Saito H, Saito T et al (1997) The incidence of apoptic bodies in the membrane granulose can predict prognosis of ova from patients participating in in vitro fertilization programs. Fertil Steril 68:312-317

44. Agarwal A, Gupta S, Sikka S (2006) The role of free radicals and antioxidants in reproduction. Curr Opin Obstet Gynecol $18: 325-332$

45. Agarwal A, Gupta S, Sekhon L et al (2008) Redox considerations in female reproductive function and assisted reproduction: from molecular mechanisms to health implications. Antioxid Redox Signal 10:1375-1403

46. Gupta S, Agarwal A, Krajcir N et al (2006) Role of oxidative stress in endometriosis. Reprod Biomed Online 13:126-134

47. Szczepanska M, Kozlik J, Skrzypczak J et al (2003) Oxidative stress may be a piece in the endometriosis puzzle. Fertil Steril 79:1288-1293

48. Saito H, Seino T, Kaneko T et al (2002) Endometriosis and oocyte quality. Gynecol Obstet Invest 53:46-51

49. Qiao J, Yeung WSB, Yao YQ et al (1998) The effects of follicular fluid from patients with different indications for IVF treatment on the binding of human spermatozoa to the zona pellucida. Hum Reprod 13:128-131

50. Aeby TC, Huang T, Nakayama RT (1996) The effects of peritoneal fluid from patients with endometriosis on human sperm function in vitro. Am J Obstet Gynecol 174:1779-1783

51. Faber BM, Chegini N, Mahony MC et al (2001) Macrophage secretory products and sperm zona pellucida binding. Obstet Gynecol 98:668-673

52. Azem F, Lessing JB, Geva E et al (1999) Patients with stage III and IV endometriosis have a poorer outcome of in vitro 
fertilization-embryo transfer than patients with tubal infertility. Fertil Steril 72:1107-1109

53. Olivennes F, Feldberg D, Liu HC et al (1995) Endometriosis: a stage by stage analysis-the role of in vitro fertilization. Fertil Steril 64:392-398

54. Arici A, Oral E, Bukulmez O et al (1996) The effect of endometriosis on implantation: results from the Yale University in vitro fertilization and embryo transfer program. Fertil Steril 66:603-607

55. Minici F, Tiberi F, Tropea A et al (2008) Endometriosis and human infertility: a new investigation into the role of eutopic endometrium. Hum Reprod 23:530-537

56. Lessey BA, Castlebaum AJ, Sawin SW et al (1994) Aberrant integrin expression in the endometrium of women with endometriosis. J Clin Endocrinol Metab 79:643-649

57. Khorram O, Lessey BA (2002) Alterations in expression of endometrial endothelial nitric oxide synthase and $\alpha v \beta 3$ integrin in women with endometriosis. Fertil Steril 78:860864

58. Hii LLP, Rogers PAW (1998) Endometriosis vascular and glandular expression of integrin $\alpha \mathrm{v} \beta 3$ in women with and without endometriosis. Hum Reprod 13:1030-1035

59. Matsuzaki S, Canis M, Darcha C et al (2009) HOXA-10 expression in the mid-secretory endometrium of infertile patients with endometriosis, uterine fibromas or unexplained infertility. Hum Reprod.2009; Advance access published:1-8

60. Hugo M, Casoy J, Filho JV (2009) Is aromatase expression in the endometrium the cause of endometriosis and related infertility? Gynecol Endocrinol 25:253-257

61. Kao LC, Germeyer A, Tulac S et al (2003) Expression profiling of endometrium from women with endometriosis reveals candidate genes for disease-based implantation failure and infertility. Endocrinilogy 44:2870-2881

62. Daftary GS, Taylor HS (2004) EMX2 gene expression in the female reproductive tract and aberrant expression in the endometrium of patients with endometriosis. J Clin Endocrinol Metab 89:2390-2396

63. Dimitriadis E, Stoikos C, Stafford-Bell M et al (2006) Interleukin-11. IL-11 receptor $\alpha$ and leukemia inhibitory factor are dysregulated in endometrium of infertile women with endometriosis during implantation window. J Reprod Immunol 69:53-64

64. Sung L, Mukherjee T, Takeshige T et al (1997) Endometriosis is not detrimental to embryo implantation in oocyte recipients. J Assist Reprod Genet 14:152-156

65. Levobic DI, Mueller MD, Taylor RN et al (2001) Immunobiology of endometriosis. Fertil Steril 75:1-10

66. Mathur S, Peress MR, Williamson HO et al (1982) Autoimmunity to endometrium and ovary in endometriosis. Clin Exp Immunol 50:259-266

67. Gleicher N, El-Roeiy A, Confino E et al (1987) Is endometriosis an autoimmune disease? Obstet Gynecol 70:115-122

68. Kennedy SH, Starkey PM, Sarget IL et al (1990) Antiendometrial antibodies in endometriosis measured by an enzyme-linked immunosorbent assay before and after treatment with danazol and nafarelin. Obstet Gynecol 75:914-918

69. Hill JA, Anderson DJ (1989) Lymphocyte activity in the presence of peritoneal fluid from fertile women and infertile women with and without endometriosis. Am J Obstet Gynecol 161:861-864

70. Fakih H, Baggett B, Holtz G et al (1987) Interleukin 1: a possible role in the infertility associated with endometriosis. Fertil Steril 47:213-217

71. Sueldo CE, KellyE ML et al (1990) Effect of interleukin-1 on gamete interaction and mouse embryo development. J Reprod Med 35:868-872
72. Hill JA, Haimovici F, Anderson DJ (1987) Products of activated lymphocytes and macrophages inhibit mouse embryo development in vitro. J Immunol 139:2250-2254

73. Akoum A, Al-Akoum M, Lemay A et al (2008) Imbalance in the peritoneal levels of interleukin-1 and its decoy inhibitory receptor type II in endometriosis women with infertility and pelvic pain. Fertil Steril 89:1618-1624

74. Deura I, Harada T, Taniguchi F et al (2005) Reduction of estrogen production by interleukin-6 in a human granulose tumor cell line may have implications for endometriosis-associated infertility. Fertil Steril 83:1086-1092

75. Iwabe T, Harada T, Terakawa N (2002) Role of cytokines in endometriosis-associated infertility. Gynecol Obstet Investig 53:19-25

76. Zhang X, Xu H, Lin J et al (2005) Peritoneal fluid concentrations of interleukin-17 correlate with the severity of endometriosis and infertility of this disorder. BJOG 112:1153-1155

77. Bersinger NA, Von Roten S, Wunder DM et al (2006) PAPP-A and osteoprotegerin, together with interleukin-8 and RANTES, are elevated in the peritoneal fluid of women with endometriosis. Am J Obstet Gynecol 195:103-108

78. Xu H, Schultze-Mosgau A, Agic A et al (2006) Regulated upon activation, normal $\mathrm{T}$ cell expressed and secreted (RANTES) and monocyte chemotactic protein 1 in the follicular fluid accumulate differently in patients with and without endometriosis undergoing in vitro fertilization. Fertil Steril 86:1616-1620

79. Morin M, Bellehumeur C, Therriault MJ et al (2005) Elevated levels of macrophage migration inhibitory factor in the peripheral blood of women with endometriosis. Fertil Steril 83:865872

80. Gmyrek GB, Sozanski R, Jerzak M et al (2005) Evaluation of monocyte chemotactic protein-1 in peripheral blood of infertile women with endometriosis. Eur J Obstet Gynecol Reprod Biol 122:199-205

81. Kabut J, Kondera-Anasz Z, Sikora J et al (2007) Levels of complement components iC $3 b, \mathrm{C} 3 \mathrm{c}, \mathrm{C} 4$ and SC5b-9 in peritoneal fluid and serum of infertile women with endometriosis. Fertil Steril 88:1298-1303

82. Lee YL, Lee KF, Xu JS et al (2004) The embryotrophic activity of oviductal cell-derived complement $\mathrm{C} 3 \mathrm{~b}$ and Ic3B, a novel function of complement protein in reproduction. J Biol Chem 279:12763-12768

83. Barcz E, Milewski L, Radomski D et al (2008) A relationship between increased peritoneal leptin levels and infertility in endometriosis. Gynecol Endocrinol 24:526-530

84. Bedaiwy MA, Falcone T, Goldberg JM et al (2006) Peritoneal fluid leptin is associated with chronic pelvic pain but not infertility in endometriosis patients. Hum Reprod 21:788-791

85. Halme J, Becker S, Wing R (1984) Accentuated cyclic activation of peritoneal macrophages in patients with endometriosis. Am J Obstet Gynecol 48:85-90

86. Ho HN, Chao KH, Chen HF et al (1995) Peritoneal natural killer cytotoxicity and $\mathrm{CD} 25+\mathrm{CD} 3+$ lymphocyte subpopulation are decreased in women with stage III-IV endometriosis. Hum Reprod 10:2671-2675

87. Mizumoto Y (1996) Changes in NK activities and TGF-beta concentration in the peritoneal cavity in endometriosis and their interaction related with infertility. Acta Obstet Gynaecol Jpn 48:379-385

88. Seli E, Arici A (2003) Endometriosis: interaction of immune and endocrine systems. Semin Reprod Med 21:135-144

89. Wang H, Gorpudolo N, Behr B (2009) The role of prolactin and endometriosis-associated infertility. Obstet Gynecol Surv 64:542-547

90. Cunha-Filho JS, Gross JL, Lemos NA et al (2002) Prolactin and growth hormone secretion after thyrotrophin-releasing hormone 
infusion and dopaminergic (DA2) blockade in infertile patients with minimal/mild endometriosis. Hum Reprod 17:960-965

91. Chung HW, Lee JY, Moon HS et al (2002) Matrix metalloproteinase-2, membranous type 1 matrix metalloproteinase, and tissue inhibitor of metalloproteinase- 2 expression in ectopic and eutopic endometrium. Fertil Steril 78:787-795

92. Huang HF, Hong LH, Yi T et al (2004) Matrix metalloproteinase 2 is associated with changes in steroid hormones in the sera and peritoneal fluid of patients with endometriosis. Fertil Steril 81:1235-1239

93. Gottsch ML, Van Kirk EA, Murdoch WJ (1985) Role of matrix metalloproteinase 2 in the ovulatory folliculo-luteal transition of ewes. Reproduction 124:347-352

94. American fertility. Revised American Fertility Society classification (1985) Fertility society. Fertil Steril 43:351

95. Marcoux S, Maheux R, Berube S (1997) Laparoscopic surgery in infertile women with minimal or mild endometriosis. N Engl J Med 337:217-222

96. Parazzini F (1999) Ablation of lesions or no treatment in minimal-mild endometriosis in infertile women: a randomized trial. Hum Reprod 14:1332-1334

97. Jacobson TZ, Duffy JM, Barlow D et al (2002) Laparoscopic surgery for pelvic pain associated with endometriosis. Cochrane Database Syst Rev 4:CD001398

98. Tulandi T, Al-Took S (1998) Reproductive outcome after treatment of mild endometriosis with laparoscopic excision and electro coagulation. Fertil Steril 69:229-231

99. Bedaiwy M, Falcone T, Katz E et al (2008) Association between time from endometriosis surgery and outcomes of in vitro fertilization cycles. J Reprod Med 53:161-165
100. Marrs RP (1991) The use of potassium-titanyl-phosphate laser for the laparoscopic removal of ovarian endometrioma. Am J Obstet Gynecol 164:1622-1626

101. Beretta P, Franchi M, Ghezzi F et al (1998) Randomized clinical trial of two laparoscopic treatments of endometriomas: cystectomy versus drainage and coagulation. Fertil Steril 70:11761180

102. Jones KD, Sutton CJG (2002) Pregnancy rates following ablative laparoscopic surgery for endometriomas. Hum Reprod 17:782785

103. Hart RJ, Hickey M, Maouris P et al (2008) Excision surgery versus ablative surgery for ovarian endometriomata. Cochrane Libr 4:1-25

104. Somigliana E, Vercellini P, Vigano P et al (2006) Should endometriomas be treated before IVF-ICSI cycles? Hum Reprod Updat 12:57-64

105. La Torre R, Montanino-Oliva M, Marchiani E et al (1998) Ovarian blood flow before and after conservative laparoscopic treatment for endometrioma. Clin Exp Obstet Gynecol 25:12-14

106. Stepniewska A, Pomini P, Bruni F et al (2009) Laparoscopic treatment of bowel endometriosis in infertile women. Hum Reprod 24:1619-1625

107. Ferrero S, Anserini P, Abbamonte LH et al (2009) Fertility after bowel resection for endometriosis. Fertil Steril 92:41-46

108. Vercellini P, Pietropaolo G, De Giorgi O et al (2006) Reproductive performance in infertile women with rectovaginal endometriosis: is surgery worthwhile? Am J Obstet Gynecol 195:1303-1310

109. Fedele L, Bianchi S, Zanconato G et al (2006) Laparoscopic excision of recurrent endometriomas: long term outcome and comparison with primary surgery. Fertil Steril 85:694-699 